\title{
The Emergence of the Multi-Cloud Era and The Death of Big Data
}

\author{
Debnath Bhattacharyya ${ }^{1}$, N. Thirupathi Rao $^{2}$ \\ ${ }^{1,2}$ Department of Computer Science and Engineering, Vignan's Institute of \\ Information Technology (A), Visakhapatnam 530049, AP, India \\ 1'debnath@gmail.com, ${ }^{2}$ nakkathiru@gmail.com
}

\begin{abstract}
Technology had growing very fast in recent years in terms of all types of technologies. Various types of trends can be observed in almost all sorts of technologies being used in various fields of the market. The utilization new technology and its trends had becoming a very interesting and making the functioning of the works smoother and easier. The utilization of such technology trends is giving good market analysis and also better business processing applications and makings. The trends of the market can be observed or understood within minutes by the utilization of such advanced technologies. In the current article, an attempt has been made to provide the details about the latest technologies and their trends in the year 2019 and their future scope of development and their utilization in the current day applications had discussed in detail.
\end{abstract}

Keywords: Big Data, Cloud era, Hadoop, Multi-cloud, Real time Analytics.

\section{INTRODUCTION}

The word big data stands for the meaning of a large volume of data that can be as both structured data and unstructured data which can be processed with various measures and applications for better results. The analysis of data is mainly concerned with various aspects for the business and its related applications and industry. The major concept or the main point of functioning will be on the basis of three v's. The three v' stands for the Velocity, volume and variety. Velocity deals with the point of travel of data to various nodes or various locations at various destinations. The main target of the modes was to send the data or to reach the data as fast as possible and can be reached to the destination with short span of time. Now a days, to achieve these sorts of points and targets, a versatile type of sensors are being sued in such a way to manage the data transfer speeds and smart meters and RFID tags are being used to process the things more faster and updated way.

The other important aspect of three v' to be considered was the volume. The volume or the amount of data being transferred or need to be transferred and should be reached safely to the end user. The requirement of sending such huge volumes of data was mainly important due to several applications and advantages. Some of them are like the business applications, data transfers between machine to machine and other modes of transfer of data. The third most important three v' was the variety of data to be processed or to be transferred. In normal cases, the applications may have the same type of data formats. But in the recent years or the recent days of technologies, several changes had been occurred or taken place in the recent technologies and had creating a various set of advantaged and other benefits. The amount of

Article history:

Received (November 12, 2018), Review Result (March 1, 2019), Accepted (June 28, 2019) 
data being transferred or to be processed is changing from time to time. In old network models, the data size to be very small. But in recent technologies, the quality of the data to be processed or to be transferred was very high. As the quality of the data contents had increased a lot, the sizes of such files all increased a lot due to the quality of the content. In earlier models, the size of an image may be in Kbs but in recent days the size of the images or the files may be in MBs to sometimes GBs. Once this sort of large volume with variety of data had sent through the network, the network infrastructures and the other models had suffered a lot.

With these important models and techniques the utility or the utilization bog data had increased and developed a lot and it is increasing day to day in a large manner or large size. The big data era is slowly reducing or becoming to end as the new technologies and new trend in the technologies are arriving. The view of processing the data in real time scenarios is getting famous day by day and it is being using by most of the customers or the people in the society in a large manner. Now a days, the big data has become one of the main source or the utility point to be considered for a revolution of technologies to be considered in the next lien of future technologies. It is supporting the current era of technologies which were growing and getting famous day to day by their applications and their source of using was like machine learning, artificial intelligence, multi clouds support models and other real time data analytics.

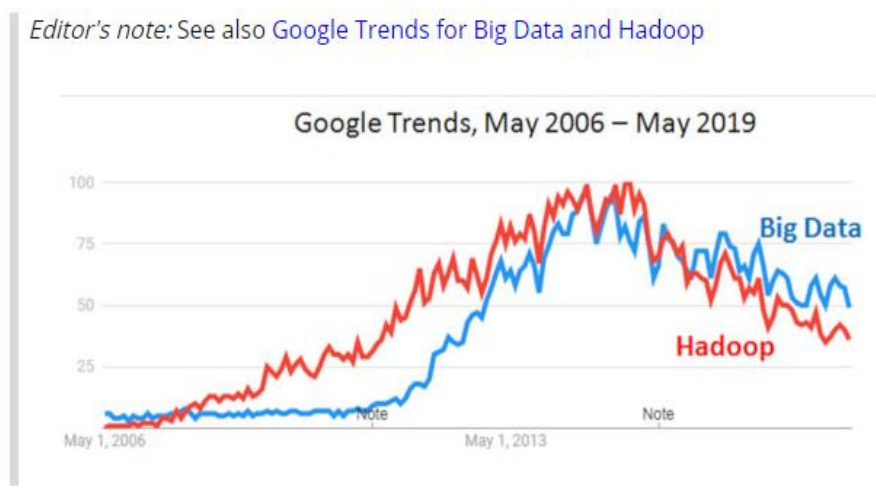

Figure.1 Google data trend analysis [1]

The trend of big data is going to an end the utilization and the focus on these areas was being shifted to the collection of data and processing of such collected data at real time scenarios or real time situations. Now a days, the big data has become one of the useful tool for the business applications and data processing applications. With this sorts of applications and other important points or things to be noted for further processing of any data related applications or the analysis of data for further more applications, the big data and its related topics and tools are further supporting the development of the further applications and their advantages. Some of the tools that may help for the better availability of the features and areas like the new areas of applications like the real-time applications, machine learning and cloud based applications [6][7].

The role of big data is going to be increased day by day as the amount of data to be processed in social media or social networking websites are increasing further a lot. As a result, the business people and the other set of people for their business applications and fir identifying the customers for their business applications is growing faster way. The role of such applications for collecting data and being submitting to those business companies or the applicants for better business opportunities are increasing a lot. The volume of data being increasing a lot such that the identification of data formats and their roles, analysis of data and 
their related concepts and the people data or customers data with various constraints also been discussed pr presented. The customer's classification can be done with various age groups and gender basis.

\subsection{THE BIRTH OF BIG DATA}

The starting or the birth of big data had started in the year of 2006 with the invention or the development of Apache Hadoop. The major goal or the utilization of this particular tool was to process or analyze the data which was in the forms of either structured data or in the form of unstructured data. The developers or the data analysts had used this software as new methods or the new techniques to analyze the data. The companies' ideology or thinking perspective on this technology changed in such a way to identify the behaviour of the customers. In general olden days, the data was thrown away or kept in the stores of data such that the data was very huge and it can be processed at very huge times. But, with the usage of such technologies, the data can be understood easily and can be processed further more easy way to analyze and understand the data trends and the customers trends for the companies for the growth of their business applications.

\subsection{CHALLENGES OF THE ENTERPRISE WORLD WITH HADOOP}

Basically the utilization of Hadoop has grown a lot for the data analytical and other analytical models applications or jobs. The major application or the advantage of using such models or these methods was that it supports a lot for the betterment or for the analysis of analyzing the large storages and analyzing of data in such storages. The major concerns or the techniques to be considered for the better processing or the better understanding of the can be supported by this model are like the ETL jobs which stands for the tasks like the Extraction of data from these storages, transforming or changing the data requirements for the better processing or to make the data more suitable for the application processing and loading the data for the further processing of the applications and their future requirements and for better understanding of the processing of such data [8].

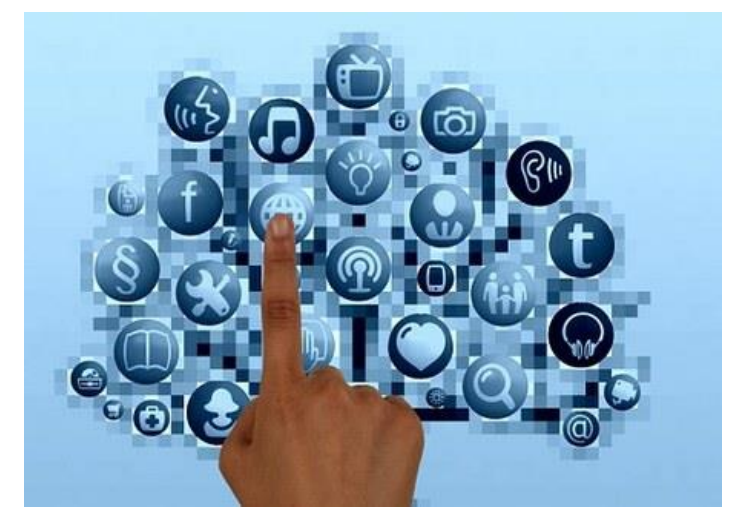

\section{Figure.2 Enterprise data model example[2]}

The Hadoop and its applications will also support the processing of data for reaching the optimal results and their analytical jobs. In this topic, both the model $\mathrm{s}$ or the issue sot be considered are like the business applications, traditional applications, data analytics models and other related operations of the companies. Several tools are available in the market to give better 
results than the existing tool or the current tool of Hadoop. Some of those tools are like the Spark, Dremel and Hive are used such that to use for better analysis of data and for better understanding of the data. In these tools working and other issues when considered, an important understanding or the important consideration had found was that the Hadoop was never faster or better performance than these applications whenever it is considered for the better understanding and for better processing of such data.

\section{APPLICATIONS AND CHALLENGES OF BIG DATA}

The Hadoop also faced several challenges from various sources of collecting and solving the problems of data analytics with databases. The main problems or challenges it had faced from the SQL and NoSQL databases and other storage providers. Basically Hadoop was designed or modelled to face such problems and try to analyze and give some solutions to the existing such data formats, but it happened reverse by not supporting fully or to some extent it is being supported for further more analysis of data. At the same time, the support of Hadoop for the business and its related applications had became a big challenge due to several reasons like the supporting business applications. The next consideration problems or the big challenges are the flexibility in adjusting or supporting several important facilities or the challenges like supporting in processing of geospatial data. Real time collected and processing of such data and currently evolving analytical data models. With the development in evolving such huge amounts of data, the Hadoop had failed to support or to process such batch processing or the processing of such huge volumes of data for further applications.

As time passes, the challenges related to various aspects for processing any databases or any other issues are increasing day to day and other issues are raising daily basis when the implementation process started. The business data and other issues were being increasing day to day and other issues are rising in various aspects. The data formats and schemes related to data models are increasing a lot day by day and the resources are increasing. Various aspects are increasing and the issues are arising due to the concerns like schemes of data,

If it seems like a lot of these companies have been in the spotlight, either from an acquisition or funding perspective, it is no coincidence. Recent examples include, but are not limited to:

ThoughtSpot's \$145 million D Round in May 2018

Sisense's $\$ 80$ million E round in September 2018

Incorta's \$15 million B round extension in October 2018

Fivetran's \$15 million A round in December 2018

Looker's \$103 million E round in December 2018

TIBCO's acquisition of Orchestra Networks in December 2018

Logi Analytics' acquisition of Jinfonet in February 2019

Google's acquisition of Alooma in February 2019

Qlik's acquisition of Attunity in February 2019

Informatica's acquisition of AllSight in February 2019

TIBCO's acquisition of SnappyData in March 2019

Alteryx' acquisition of ClearStory Data in April 2019

Matillion's \$35 million C round in June 2019

Google's intent to acquire Looker in June 2019

Salesforce's intent to acquire of Tableau in June 2019

Logi Analytics' acquisition of Zoomdata in June 2019

With the Era of Big Data coming to an end, we now can focus less on the mechanics of collecting large volumes of data and more on the myriad challenges of processing, analyzing, 
and interacting with massive amounts of data in real-time. Here are a few concepts to keep in mind as we progress to new Eras driven by Big Data.

Some of the new eras that big data has its impact on the growth of some new technologies are like.

\subsection{HADOOP STILL HAS ITS PART IN ENTERPRISE DATA}

The researchers and the experts had a thought or an idea of introducing the MapR model methods can make or bring an end to the technologies that IT companies are being looking for the several other models like the CA or Micro Focus. The researchers and other users had thought that the Cloudera had took several other steps such that to control the flow and the development of this models. As a result, the researchers had thought and believed that the MapR can dominate the Cloudera and can override all the facilities.

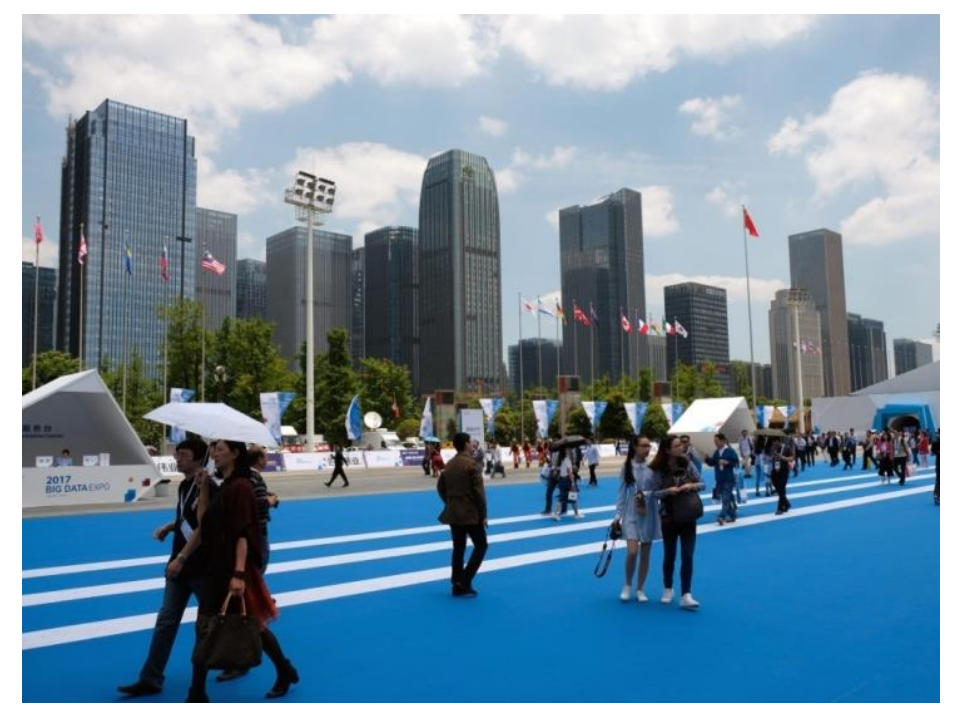

Figure.3 Enterprise model example placed in China [3]

Cloudera has taken some sufficient time to change from its regular form of models to the other forms of the transformations with in the decades based on some time frames. Most of the successful companies had already a prepared and some other companies are ready to go for the transformation of the technologies with some time frames.

\subsection{THE DAY TO DAY NEED FOR DATA VISUALIZATION AND MULTI CLOUD ANALYTICS IS INCREASING A LOT}

In the process of development in the area of data visualizations and data cloud analytics, several big companies had tried a lot and acquired so many other companies working on these areas of data analytics. The companies which come under this mode are like the Google and Salesforce are best examples of these sorts of procedures. These two companies had recently acquired two other big giants in the field of data analytics and other cloud data analytics are like the Tableau and Looker companies which was an worth of 18 billion dollars. The growth of the requirements and business on these areas are growing in such a large scale in the current day market. The tasks which were related with the multi-cloud analytics are like the processing 
of data, fragmenting the data and storage of data, computing and integration of such huge data are some of the important tasks to be performed by these most of these companies.

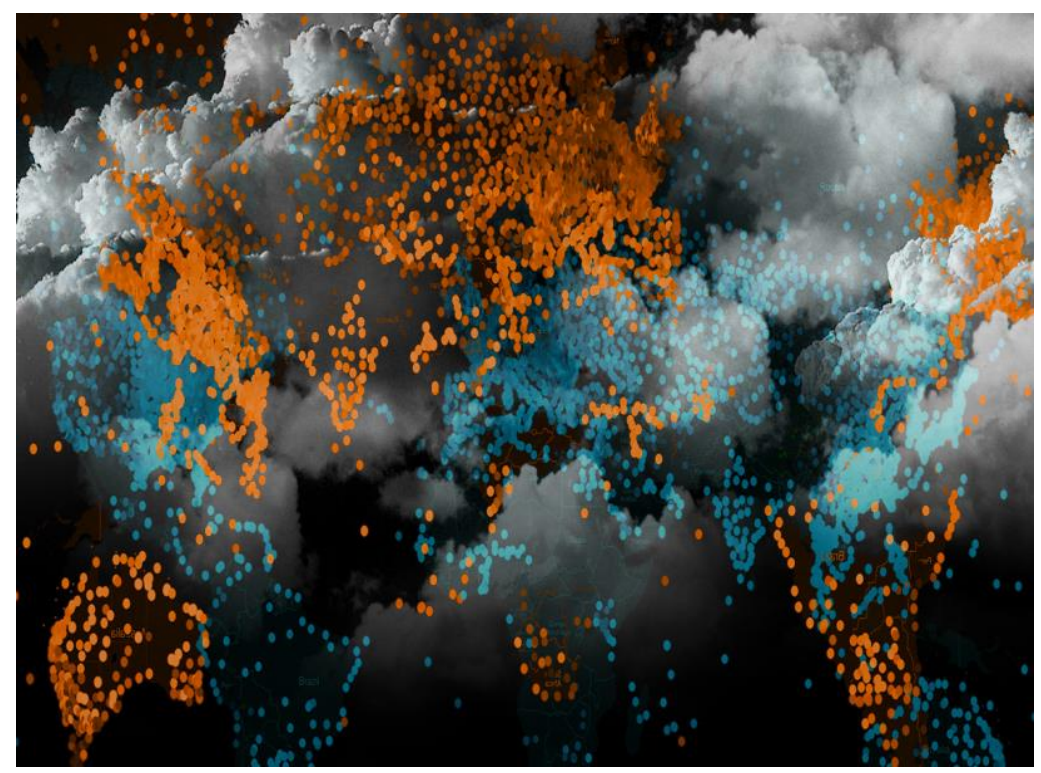

Figure.4 Multi Cloud Analytics model example [4]

The companies need to work more and more for better results and for better outputs from these data storage models. The enterprise data is being increasing in large scale day to day and the need of analyzing such data is also growing in a faster way such that to monitor the process of such data. The processes that can be performed on such data formats or the data storages are like the data modelling, data integration, data analysis, data analytics, machine learning models, data science models, data science teams, models, data analysts, complex data problems etc., can be resolved.

\subsection{DATA SCIENCE AND MACHINE LEARNING BASED ANALYTICAL ANALYSIS AND DATA MANAGEMENT EFFORTS.}

For performing several applications and tasks, some special efforts and support from some special models are required. Some of the tasks where the need of requirements will be raised are like testing of data, synthesis of data, distributions of parameters and assumptions based on algorithms etc., need the support for special efforts and other models based definitions. Some big data special assumptions are required for the better applications and better outputs from the sources or from the traditional efforts. The most interesting and important consideration was the usage of data that may not be used by others or also to use such data that can be used for better market analysis and better outputs from such units. The points to be considered for such issues are like the lack of sources for data analysis, defining data poorly, conceptualizing the data very much poorly, and size of the samples in very small size, algorithmic assumptions and classification models. 


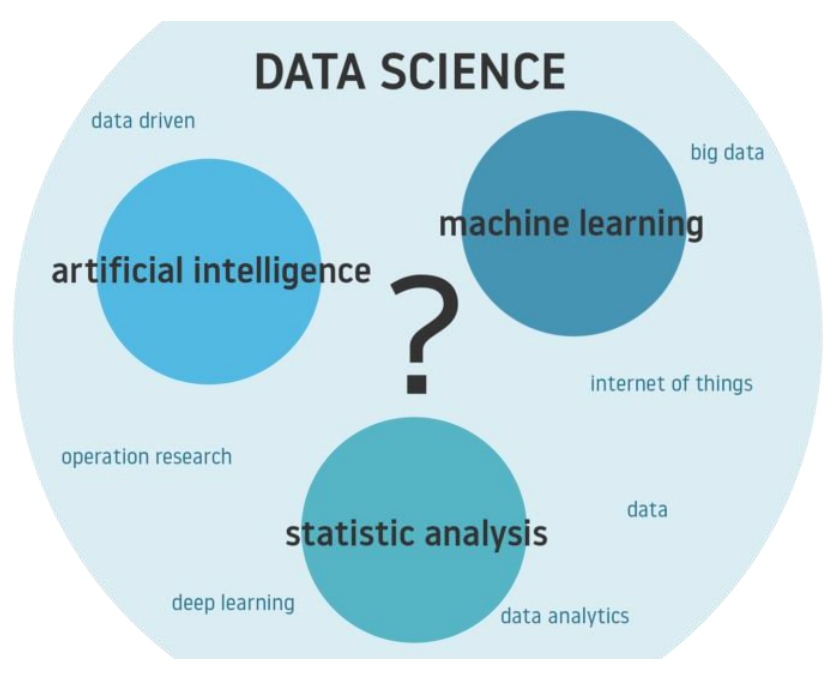

\section{Figure.5 Machine learning model analytics example}

The same concept can be observed and viewed in a different way of mode like the data that was already available and data that cannot be used should be used in the current context. The existing data may give raise to several issues like the biased models, inaccurate models, destruction of the models etc. The artificial intelligence is one of the most prominent and most utilized technologies for solving such sorts of issues and tries to give some solutions to these sorts of problems. The machine learning was one of the advanced applications of artificial intelligence and its application based algorithms will help us to deal seriously with such problems and tries to deal and solve such issues related to those sorts of models. This model of applications and its related topics can be monitored and managed, governed and supported in a critical way such that to solve such issues.

\subsection{UBIQUITOUS AND REAL TIME CONTEXT NEEDS TO BE SEEN AS TECHNOLOGICAL AND COLLABORATIVE CHALLENGES.}

The world is going so fast and developing in such a faster manner where the technology is playing a vital role in almost all the steps of the life of people in the society. The technology is moving faster and with the same technology the users and the customers also need to be accessed and worked with such models accurately and fastly. Almost all the companies in the worlds are behind this model of methods and always tries to collect as more number of customers data and their likes and tastes and base don't he analysis of such customers data, the companies tries to contact the customers in various forms of the methods. Some of the ways that companies will try to contact or try to influence the customers or can attract the customers are like giving advertisements to their mails, displaying on their mobile phones or playing adds on their mobile phones or other applications they are using and also they send their updates or increase of reduce on prices to customers randomly base don't he interests of the customers. It is also the fact that the companies will try almost all the contact methods to reach to customers in all means such that to reach the actual customers. It is very difficult to escape from all means of advertisements that these companies are sending such advertisements to the actual customers.

Some of the companies that were performing such type of advertisements and data collection of customers and reaching to customers in various means are several in industry. The actions of each and every user can be watched, monitored and the action can be recorded for further 
processing and for better analysis of data. The size of such data was increasing a lot day by day and the concept of these tasks performance was getting slow and slow. The users are depending on their choice, the purchasing or the suspension of such data can be suspended or preceded for the further processing of such data and its related applications.[5]

\section{CONCLUSION}

In the current article an attempt has been made to provide a detailed analysis on the aspect of era of big data has been to an end and the new birth or the usage if such methods to the other methods. But, in the way of identifying such methods and models, the bigdata itself has become as part of such new methods more effectively and playing a key role in the process of such methods implementations and processing. The bigdata has become one of the core aspects of such area with a new bright future. The Bigdata has to be used now days in the aspect of new tool or new methods mode for business applications and its related applications. The new trends and areas, how the big data can be used as a good tool for bright future had discussed and given in detail.

\section{References}

[1] Hyoun Park, "The Death of Big Data and the Emergence of the Multi-Cloud Era”, KDnuggets Home, (2019)

[2] https://www.likethetruth.com/big-data.html (2019)

[3] https://www.kpvu.org/post/remote-chinese-province-uses-its-climate-grow-big-data-industry (2019)

[4] https://www.wandisco.com/news-events/blog/tech-and-trends/multi-cloud-futures-are-driving-evolved-datastrategies-cloud (2019)

[5] https://datascience.aero/artificial-intelligence-vs-data-science/(2019)

[6] Ruimin Hu et. Al., "Key Technology for Big Visual Data Analysis in Security Space and Its Applications", 2016 International Conference on Advanced Cloud and Big Data (CBD), 13-16 Aug. (2016), [DOI: 10.1109/CBD.2016.065]

[7] Jian Yin et. Al., "Data confidentiality challenges in big data applications", 2015 IEEE International Conference on Big Data (Big Data)”, 29 Oct.-1 Nov. (2015), [DOI: 10.1109/BigData.2015.7364111]

[8] B. Kezia Rani et. Al., "Scheduling of Big Data application workflows in cloud and inter-cloud environments", 2015 IEEE International Conference on Big Data (Big Data), (2015) [DOI: 10.1109/BigData.2015.7364103] 